\title{
Variation in Laboratory Utilization and Correlation with Hospital Bed Utilization: Experience of a Trauma- Care Hospital during the COVID-19 Pandemic
}

\author{
Tapasyapreeti Mukhopadhyay ${ }^{10}$ Narinder Kumar ${ }^{2}$ \\ Nirupam Madaan ${ }^{2}$ Rajesh Malhotra ${ }^{4}$

\footnotetext{
${ }^{1}$ Department of Laboratory Medicine, Jai Prakash Narayan Apex Trauma Centre, All India Institute of Medical Sciences, New Delhi, India

2 Department of Hospital Administration, Jai Prakash Narayan Apex Trauma Centre, All India Institute of Medical Sciences, New Delhi, India

${ }^{3}$ Department of Biostatistics, All India Institute of Medical Sciences, New Delhi, India
}

J Lab Physicians 2022;14:210-217.

\author{
Shivam Pandey ${ }^{3}$ \\ Arulselvi Subramanian ${ }^{10}$
}

\author{
Address for correspondence Arulselvi Subramanian, MD, \\ Department of Laboratory Medicine, Jai Prakash Narayan Apex Trauma \\ Centre, All India Institute of Medical Sciences, New Delhi 110029, \\ India (e-mail: arulselvi.jpnatc@gmail.com). \\ ${ }^{4}$ Department of Orthopedics, Jai Prakash Narayan Apex Trauma \\ Centre, All India Institute of Medical Sciences, New Delhi, India
}

\author{
Abstract \\ Keywords \\ - bed occupancy \\ - hospital information \\ system \\ - biosafety \\ - laboratories \\ - pandemic \\ - SARS-CoV-2 \\ - traumatology
}

Objectives The present study was planned with the following objectives: (i) to calculate the difference in frequency of laboratory test ordered and use of consumables between the prepandemic and pandemic phases, (ii) to determine and compare the monthly average number of tests ordered per patient between the prepandemic and pandemic phases, and (iii) to correlate the monthly test ordering frequency with the monthly bed occupancy rate in both phases.

Materials and Methods Records of laboratory tests ordered and use of consumables were collected for the prepandemic phase (1.8.2019 to 31.3.2020) and the pandemic phase (1.4.2020 to 31.10.2020). The absolute and relative differences were calculated. Monthly average number of tests ordered per patient and bed occupancy rate between prepandemic and pandemic phases was determined, compared, and correlated.

Statistical Analysis The absolute and the relative differences between the two periods were calculated. The continuous variables were analyzed between groups using Mann-Whitney $U$ test. Spearman correlation was used to correlate the monthly test ordering frequency with the monthly bed occupancy rate in both phases.

Results A total of 946,421 tests were ordered, of which 370,270 (39\%) tests were ordered during the pandemic period. There was a decrease in the number of the overall laboratory tests ordered (12\%), and in the use of blood collection tubes (34\%), and an increase in the consumption of sanitizers (18\%), disinfectants (3\%), masks (1633\%), and gloves (7011\%) during the pandemic period. Also, the monthly average number of tests ordered per patients significantly reduced ( $p$-value $<0.001$ ). Test ordering frequency published online November 17, 2021
DOI https://doi.org/ $10.1055 / \mathrm{s}-0041-1739540$. ISSN 0974-2727. (c) 2021. The Indian Association of Laboratory Physicians. All rights reserved.

This is an open access article published by Thieme under the terms of the Creative Commons Attribution-NonDerivative-NonCommercial-License, permitting copying and reproduction so long as the original work is given appropriate credit. Contents may not be used for commercial purposes, or adapted, remixed, transformed or built upon. (https://creativecommons.org/ licenses/by-nc-nd/4.0/)

Thieme Medical and Scientific Publishers Pvt. Ltd., A-12, 2nd Floor, Sector 2, Noida-201301 UP, India 
had strong positive correlation with bed occupancy rate during pandemic (Spearman co-efficient $=0.73$, $p$-value $=0.03$ ).

Conclusions An overall decline in laboratory utilization during pandemic period was observed. Understanding and correlating the trends with hospital bed utilization can maximize the productivity of the laboratory and help in better preparedness for the challenges imposed during similar exigencies.

\section{Introduction}

On March 24, 2020, a nationwide lockdown was announced in the country to manage the coronavirus disease 2019 (COVID19) pandemic. Many hospitals were converted to COVID-19 care centers due to the anticipated rise in the number of cases. As the pandemic evolved, the previously unrecognized and unprecedented role of a diagnostic laboratory in patient care also unfolded. ${ }^{1,2}$ It was a challenge for the laboratories in the converted hospitals to cater efficiently to the new requirements to maintain the highest standards of care for COVID-19 patients. The challenges that the laboratories faced included formulation and execution of modified protocols for biosafety, specimen collection, transport, processing, analyzing, and prioritizing important steps. New test parameters and testing platforms were also introduced in accordance with the new patient population to assure that patient services were of the highest quality while ensuring safety.

Regardless of the size of a clinical laboratory, a fair idea of the prevalent or anticipated test ordering pattern due to the changing trends in hospital bed utilization is vital to ensure quality patient care. Different issues may arise when faced by an emergency like the current pandemic, when there is a sudden change in test demands and use of consumables due to the changed patient population, average laboratory test ordered per patients, and the bed occupancy rate.

This study was planned with the following primary objectives: (i) to calculate the difference in frequency of laboratory test ordered and use of consumables between the prepandemic and pandemic phases, (ii) to determine and compare the monthly average number of tests ordered per patient between the pre-pandemic and pandemic phases; and the secondary objective (iii) to correlate the monthly test ordering frequency with the monthly bed occupancy rate in both phases.

\section{Materials and Methods}

This retrospective study was conducted in a central laboratory of a 250-bedded apex trauma center at a tertiary care hospital. The hospital was declared as a COVID-19 care center on March 23, 2020. During the prepandemic period, the laboratory catered to both inpatients and outpatients, although only a small proportion of outpatients required laboratory tests. However, during the pandemic, hospital outpatient services were halted and subsequently, laboratory services were limited to the inpatients.
Laboratory tests including complete blood count, coagulation profile, biochemical examination, immunology, and histopathology examination are being performed in the laboratory. However, the new test parameters (interleukin-6, ferritin, procalcitonin, and lactate dehydrogenase) relevant to the therapeutic monitoring of COVID-19 were introduced on May 26, 2020 in the laboratory. The instrument Beckman Coulter Access 2 was installed for the purpose. Subsequently, the medical technologists who were supposed to operate the instruments were trained about the related test operations and quality assurance. The healthcare professionals were trained regarding the test selection templates newly introduced on the laboratory information system. The initial training was done over 2 days, in small groups of two or three people in the laboratory, maintaining the norms of social distancing. Subsequently, assistance on troubleshooting and machine maintenance was provided via audio/video calls by the instrument engineer. This was applicable for the previously installed instruments in the laboratory as well.

The entire study period was divided into (i) the prepandemic phase from August 1, 2019 to March 31, 2020, and (ii) the pandemic phase from April 1 to November 30, 2020. Between March 23, 2020 (the day the hospital was declared as a COVID-19 care center), and March 31, 2020, the hospital and the laboratory observed changes in logistics related to the general preparedness for the management of COVID-19 patients. The hospital admissions were minimal, resulting in negligible ordering of tests and use of laboratory consumables during this period. Thus, for convenience, the census related to this period has been included in the prepandemic period.

The healthcare professionals order laboratory tests through the hospital information system (e-Hospital ${ }^{\circledR N I C}$ ). On completion of the test requested, the results are uploaded by the medical technologists working in the laboratory. The results can be viewed by the healthcare professionals at the patient site only after they are validated by a laboratory physician or the laboratory in-charge.

For the study, the frequency of the following tests was noted: blood glucose, urea, creatinine, calcium, phosphate, uric acid, sodium, potassium, total bilirubin, direct bilirubin, indirect bilirubin, total protein, albumin, globulin, alanine transaminase, aspartate transaminase, alkaline phosphatase, amylase, magnesium, cholesterol, triglycerides, high-density lipoproteins, low-density lipoproteins, very low-density lipoproteins, complete blood count, peripheral blood smear 
examination, prothrombin time, activated partial thromboplastin time, D-dimer, and the immune biomarkers for COVID-19 care namely C-reactive protein (CRP), procalcitonin, ferritin, interleukin-6, and lactate dehydrogenase. The records of microbiological tests were excluded from the study.

Data related to the use of consumables notably, blood collection tubes (BCT) (Becton Dickinson (BD) Franklin Lakes, New Jersey, United States), alcohol-based sanitizers, surface disinfectants, face masks, gloves, personal protective equipment (PPE), disposable cap, and shoe covers used in the laboratory were also noted from the online indent system for the two phases.

The average frequency of laboratory tests ordered per month and monthly average laboratory tests ordered per patient was determined for the two phases and compared. The monthly average number of laboratory tests ordered per patient was calculated using the following formula: Monthly average number of laboratory tests ordered per patient = Total number of laboratory tests ordered in a month/number of inpatients in that month.

The data related to the bed occupancy rate and number of patients hospitalized per month were collected using the hospital information system. The bed occupancy rate was calculated using the following formula and compared for the two phases: average bed occupancy rate $=[$ Total number of inpatient days for a given period/(Total available beds $x$ Number of days in the period)] $\times 100$.

Furthermore, the monthly test ordering frequency was correlated with the monthly bed occupancy rate in both phases.

Approval from the Institute Ethics Committee was obtained prior to conducting this study (IEC-203/09.04.2021).

\section{Statistical Analysis}

Data collected is descriptive and is represented in percentages. The absolute and the relative differences in the laboratory test ordered and the consumables used between the two periods were calculated. The continuous variables were analyzed between groups using Mann-Whitney $U$ test. Spearman correlation was used to correlate the monthly test ordering frequency with the monthly bed occupancy rate in both phases. The $p$-value less than 0.05 was considered significant. Data was analyzed using StataCorp. 2017 (Stata Statistical Software: Release 15. College Station, StataCorp LLC, Texas, United States).

\section{Results}

\section{Variation in Laboratory Test Ordering Frequency}

There were total of 946,421 laboratory tests ordered and verified during the entire study period, of which 576,151 (61\%) and 370,270 (39\%) belonged to the prepandemic and the pandemic phases, respectively. During the pandemic phase, there was a decrease in overall test ordering frequency by $36 \%$. The frequency and the absolute and relative differences for each test parameter ordered in the prepandemic and pandemic period are shown in - Table 1. Monthly laboratory test ordering frequency during the pandemic period is shown in - Fig. 1. The overall monthly laboratory test ordering frequency during the pandemic was 46,443 and was significantly lower than the prepandemic phase shown in -Table $2(p$-value $=0.020)$.

\section{Variation in Laboratory Test Ordering Pattern}

Complete blood count (6.1\%) was the most ordered test in the prepandemic phase in contrast to creatinine (5.1\%) being the most ordered parameter during the pandemic phase as shown in - Table 1. New test parameters namely ferritin, procalcitonin, and interleukin- 6 and lactate dehydrogenase were introduced during the pandemic phase and constituted $2.6 \%$ of all the ordered laboratory test parameters. A notable increase in test ordering of CRP (100\%), magnesium (55\%), and D-dimer (12405\%) was observed during the pandemic.

\section{Variation in Use of Consumables}

A total of 123,052 BCT were used during the entire study duration of which 49,639 (40.3\%) were used during the pandemic phase. There was $32 \%$ decrease in the overall use of BCT during the pandemic phase. The absolute and relative differences in the frequency of use of each type of BCT used in the prepandemic and pandemic period have been shown in - Table 3. During the pandemic, there was $18 \%$ and $3 \%$ increase in the consumption of alcohol-based sanitizers and surface disinfectants, respectively. The use of disposable face masks (1633\%) and disposable gloves (7011\%) also increased during the pandemic.

\section{Variation in Monthly Average Number of Laboratory Tests Ordered Per Patients}

The monthly average number of laboratory tests ordered per patient was 111 in the prepandemic period and it significantly reduced to 59 during the pandemic ( $p$-value $<0.001)$. - Table 2 shows the overall frequency of the laboratory tests ordered and the number of inpatients admitted in that month during the entire study period and in each month.

\section{Correlation of Monthly Test Ordering Frequency with the Monthly Bed Occupancy Rate in the Prepandemic and Pandemic Phases}

The monthly test ordering frequency did not correlate with the monthly bed occupancy rate in the prepandemic phase (Spearman co-efficient $=-0.09, p$-value $>0.05$ ). In contrast, the monthly test ordering frequency had a strong positive correlation with the monthly bed occupancy rate in the pandemic phase $($ Spearman co-efficient $=0.73, p$-value $=0.03$ ). - Table 2 shows the overall bed occupancy rate during the entire study period and for each month.

\section{Discussion}

The first major finding of this study was that there was an overall decline in the laboratory test ordering frequency. The initial decline in the frequency of laboratory test ordering was drastic due to the overall lower number of COVID-19 
Table 1 Difference in the frequency of laboratory test ordered between the prepandemic and the pandemic period

\begin{tabular}{|c|c|c|c|c|}
\hline Tests ordered & Prepandemic & During pandemic & Absolute difference & Relative difference \\
\hline Urea & 31,829 & 18,585 & $-13,244$ & -42 \\
\hline Creatinine & 32,679 & 19,039 & $-13,640$ & -42 \\
\hline Calcium & 32,152 & 19,035 & $-13,117$ & -41 \\
\hline Phosphate & 31,866 & 19,030 & $-12,836$ & -41 \\
\hline Uric acid & 31,928 & 19,020 & $-12,908$ & -41 \\
\hline Sodium & 32,850 & 19,030 & $-13,820$ & -42 \\
\hline Potassium & 32,850 & 19,030 & $-13,820$ & -42 \\
\hline Total bilirubin & 28,427 & 19,033 & $-9,394$ & -33 \\
\hline Direct bilirubin & 28,427 & 19,033 & $-9,394$ & -33 \\
\hline Indirect bilirubin & 28,427 & 19,033 & $-9,394$ & -33 \\
\hline Total protein & 27,516 & 18,994 & $-8,522$ & -31 \\
\hline Albumin & 27,516 & 18,994 & $-8,522$ & -31 \\
\hline Globulin & 27,516 & 18,994 & $-8,522$ & -31 \\
\hline AST & 27,516 & 18,993 & $-8,523$ & -31 \\
\hline ALT & 27,516 & 18,991 & $-8,525$ & -31 \\
\hline ALP & 27,516 & 18,991 & $-8,525$ & -31 \\
\hline Amylase & 3,913 & 1,759 & $-2,154$ & -55 \\
\hline Glucose & 9,698 & 1,473 & $-8,225$ & -85 \\
\hline Cholesterol & 4,691 & 1,882 & $-2,809$ & -60 \\
\hline Triglycerides & 630 & 496 & -134 & -21 \\
\hline $\mathrm{HDL}$ & 630 & 496 & -134 & -21 \\
\hline LDL & 630 & 496 & -134 & -21 \\
\hline VLDL & 630 & 496 & -134 & -21 \\
\hline CRP & 1,963 & 3,934 & 1,971 & 100 \\
\hline Interleukin-6 & 0 & 2,251 & 2,251 & - \\
\hline Ferritin & 0 & 4,448 & 4,448 & - \\
\hline Procalcitonin & 0 & 3,091 & 3,091 & - \\
\hline $\mathrm{LDH}$ & 0 & 1,057 & 1,057 & - \\
\hline Magnesium & 837 & 1,299 & 462 & 55 \\
\hline $\mathrm{CBC}$ & 35,229 & 18,960 & $-16,269$ & -46 \\
\hline Peripheral smears & 2,053 & 1,712 & -341 & -17 \\
\hline PT & 19,349 & 10,661 & $-8,688$ & -45 \\
\hline APTT & 19,349 & 10,661 & $-8,688$ & -45 \\
\hline D-dimer & 18 & 2,251 & 2,233 & 12,405 \\
\hline Total tests & 576,151 & 370,270 & $-205,881$ & -36 \\
\hline
\end{tabular}

Abbreviations: ALP, alkaline phosphatase; ALT, alanine transaminase; APTT, activated partial thromboplastin time; AST, aspartate transaminase; CBC, complete blood count; CRP, C-reactive protein; HDL, high-density lipoproteins; LDH, lactate dehydrogenase; LDL, low-density lipoproteins; PT, prothrombin time; VLDL, very low-density lipoproteins.

cases in the region and a smaller number of hospitalized patients. Gradually, the laboratory test demand increased that paralleled the increase in the incidence of COVID-19 cases in the region as illustrated in -Fig. 2. ${ }^{3}$ Similar findings have been reported by others. ${ }^{4,5}$

The second major finding was that the complete blood count was the most ordered laboratory test in the prepan- demic phase in contrast to creatinine during the pandemic phase. And the demand for CRP, magnesium, D-dimer, interleukin-6, procalcitonin, ferritin, and lactate dehydrogenase (LDH) during the COVID-19 pandemic increased. The pattern of laboratory test ordering in a hospital setting is largely dependent on the type of patients being served. To explain further, CRP is being used as an early biomarker of innate 
214 Laboratory Utilization and the Pandemic Mukhopadhyay et al.

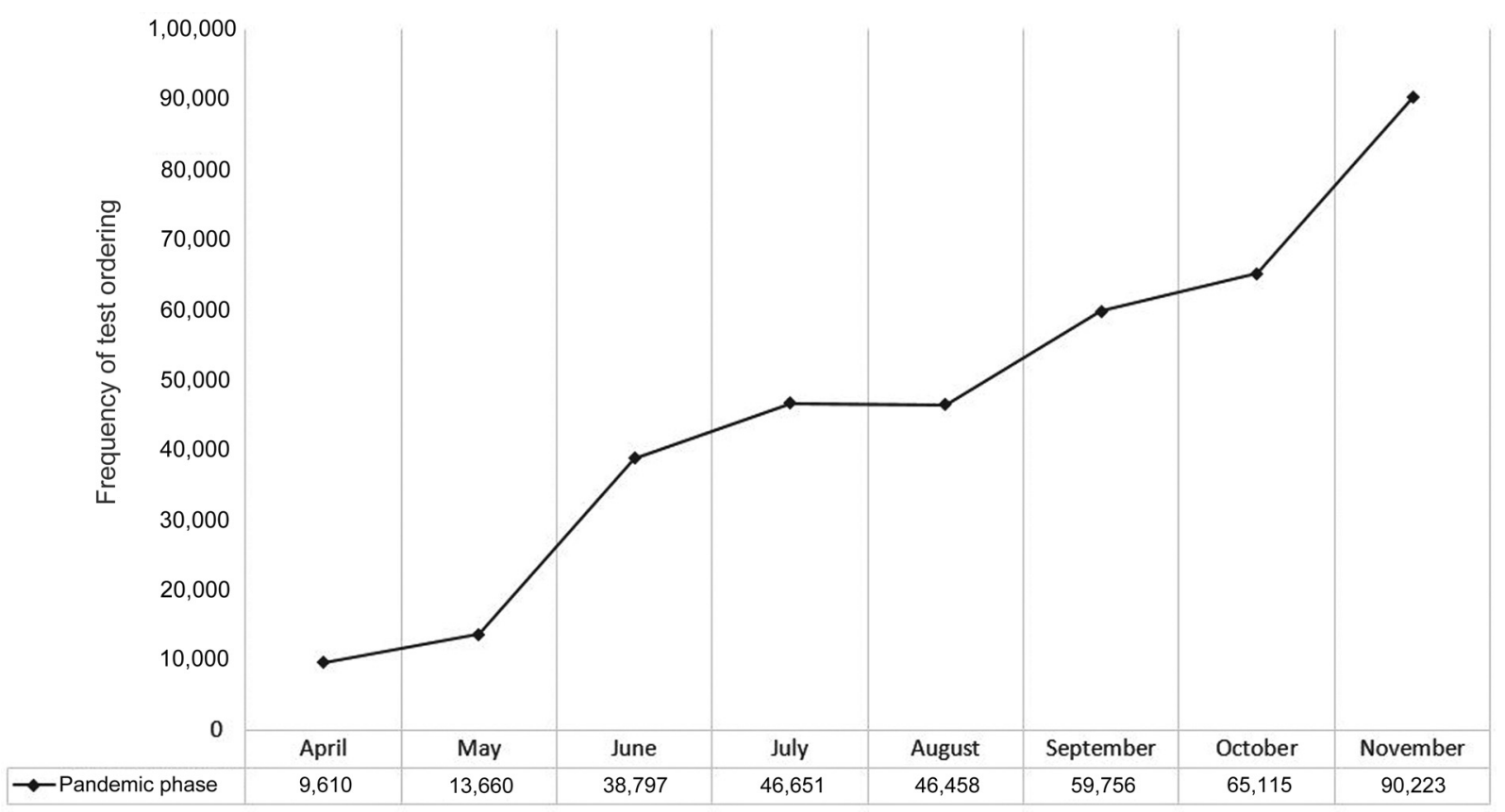

Fig. 1 Monthly frequency of laboratory test ordered during the pandemic.

immune reaction, and also a predictor for disease severity due to the severe acute respiratory syndrome coronavirus 2 (SARS-CoV-2) infection. ${ }^{6-8}$ Similarly, magnesium deficiency may lead to a weak immune system, increased susceptibility, and disease severity and thus it is monitored in COVID-19 patients. $^{9,10}$ D-dimer also correlates with disease severity and is a helpful marker used in the management of COVID-19 patients. $8,11-13$ The increased demand for procalcitonin,

Table 2 Comparison of prepandemic and pandemic periods based on laboratory utilization and hospital bed utilization

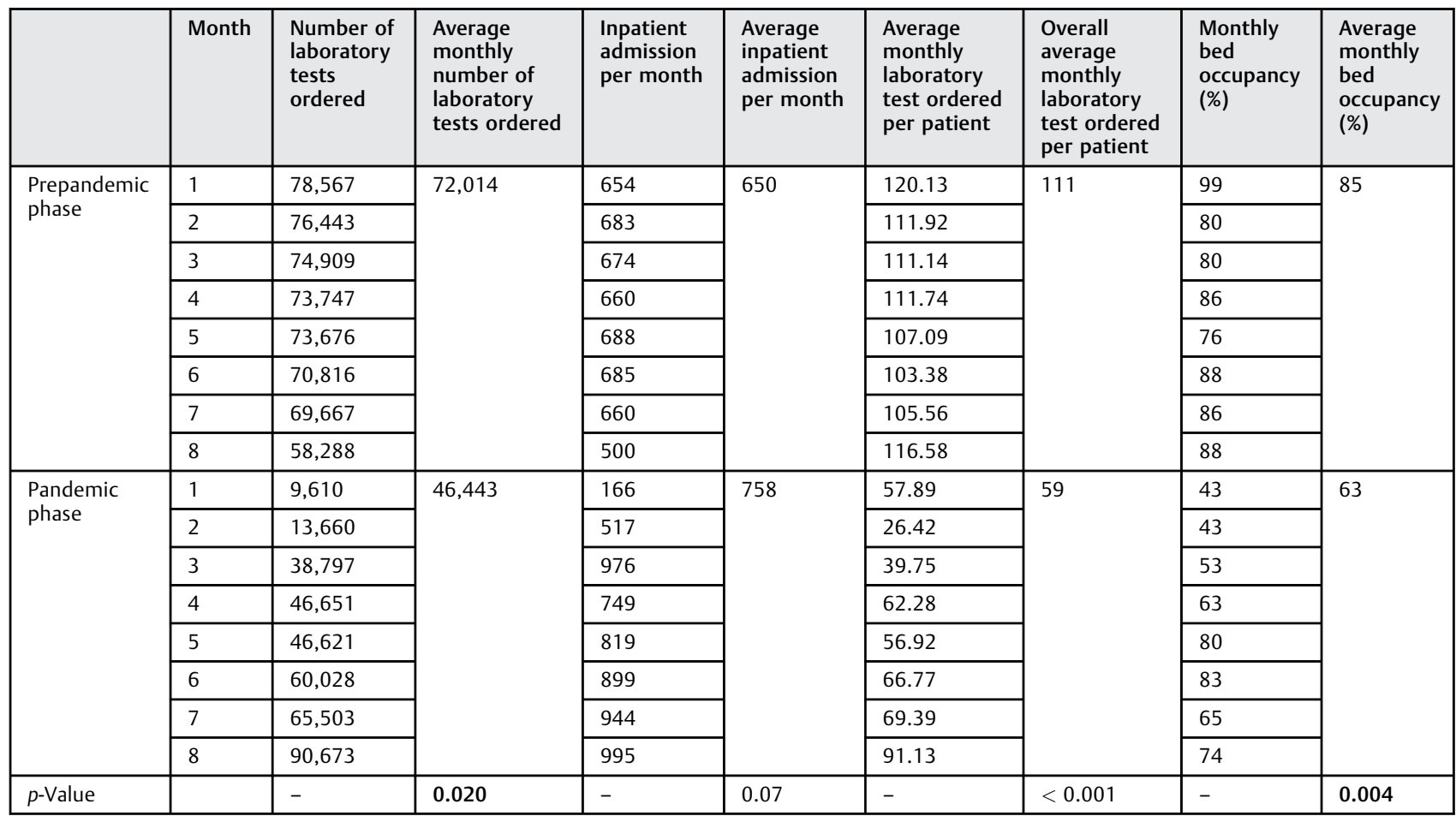

Level of significance: $p<0.05$. 
Table 3 Difference in the frequency of consumables used between the prepandemic and the pandemic period

\begin{tabular}{|l|l|l|l|l|}
\hline Specimen collection containers & Prepandemic & During pandemic & Absolute difference & Relative difference \\
\hline Blood collection tube & 73,413 & 49,639 & $-23,774$ & -32 \\
\hline$\cdot$ BCT with EDTA salt & 27,620 & 15,488 & $-12,132$ & -44 \\
\hline$\cdot$ BCT with sodium citrate & 14,252 & 9,547 & $-4,705$ & -33 \\
\hline$\cdot$ SST BCT & 24,346 & 23,131 & $-1,215$ & -5 \\
\hline • Fluoride BCT & 7,195 & 1,473 & $-5,722$ & -80 \\
\hline Alcohol-based sanitizer & 250 & 295 & 45 & 18 \\
\hline Surface disinfectant & 380 & 390 & 10 & 3 \\
\hline Face mask & 300 & 5,200 & 4,900 & 1,633 \\
\hline Gloves & 1,800 & 128,000 & 126,200 & 7,011 \\
\hline Gown & 0 & 420 & 420 & - \\
\hline PPE & 0 & 628 & 628 & - \\
\hline Cap & 0 & 4,600 & 4,600 & - \\
\hline Shoe cover & 0 & 4,800 & 4,800 & - \\
\hline
\end{tabular}

Abbreviations: BCT, blood collection tube; EDTA, ethylene diamine tetra acetic acid; PPE, personal protective equipment; SST, serum separator tube.

ferritin, and LDH is explained by their role in predicting outcome in COVID-19 infection. ${ }^{8,14,15}$ Interleukin-6 is the best available biomarker for assessing severity of COVID-19 and guides treatment. ${ }^{16}$

The laboratory experienced serious time lag in offering the immune markers for the patients. Owing to issues related to delays in supply chain because of repeated extension of nationwide lockdown, it took over 2 months to complete the formalities of purchase of the test reagents and the equipment and its installation.
The exigency prompted timely indentation of goods that ensured uninterrupted quality laboratory services, without compromising the safety of the laboratory personnel. Since a comprehensive list of all the stock with the details of their date of expiry was already being maintained as a general practice even before the pandemic started, it helped in easily identifying the consumables with dates closer to their expiry to be used at first. It also helped to promptly identify and plan the reagents and consumables to be ordered subsequently. Thus, by just maintaining a regular stock register it is

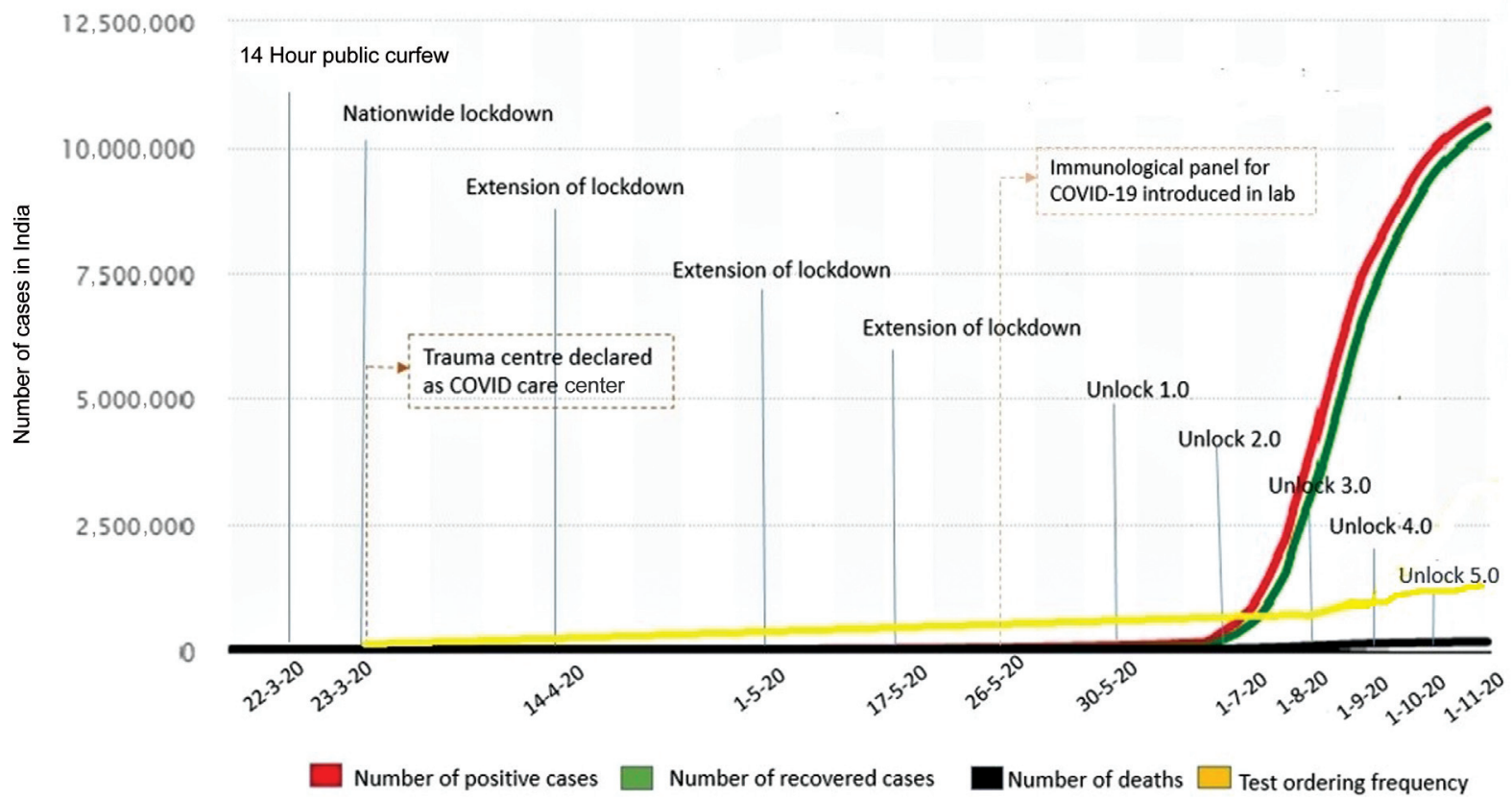

Fig. 2 Test ordering frequency and the significant events in the clinical laboratory in relation to rise in coronavirus disease 2019 (COVID-19) cases in the country (diagram not up to the scale). ${ }^{3}$ 
possible to reduce wastage of resources that is crucial in these times of emergencies, when the supply chain is compromised due to nationwide lockdown.

The third major finding of this study was the overall decline in use of BCT during the pandemic and as is intuitive, it mirrored the trend observed in case of laboratory tests ordered. Likewise, the BCT with potassium salt of ethylene diamine tetra acetic acid was commonly used during the prepandemic phase since it provided vital information related to the presence of anemia and thrombocytopenia often used to assess the need for blood transfusion in trauma patients. ${ }^{17}$ In contrast, during the COVID-19 pandemic, there was an increase in the test demand of creatinine followed by calcium and the immune biomarkers, thus requiring more volume of serum and, hence, the serum separator tube with silica clot activator biomarkers was consumed more.

The manifold increase in use of face masks, gloves, PPE, sanitizers, and surface disinfectants was also observed. Obviously, the stringent laboratory protocols to mitigate biohazard risks during the pandemic have resulted in increased consumption of these items in our set-up. ${ }^{18}$

The fourth major finding was that the monthly average number of laboratory tests ordered per patient significantly reduced during the pandemic. And, the fifth major finding was that the monthly test ordering frequency that did not correlate with the monthly bed occupancy rate in the prepandemic phase had a strong positive correlation with the monthly bed occupancy rate during the pandemic phase. The higher bed occupancy rate and the lower inpatient admissions during the prepandemic phase together suggest that during trauma care, frequent sampling was done for the patients. However, during the pandemic, although inpatient admission over the same time period was higher, the tests ordered per patient were lower and strongly correlated with bed occupancy rate, together suggesting limited laboratory test being ordered, probably to decrease the exposure of healthcare professionals to SARS-CoV-2 virus during phlebotomy.

Globally, over $10 \%$ of clinical biochemistry laboratories have restricted their test menu due to limited resources. ${ }^{19}$ However, the decline in both laboratory test ordering frequency and use of consumables along with decrease in laboratory tests ordered per patient in our setting was not only due to limited resources but perhaps also due to the general biosafety protocols adopted in the hospital to decrease the exposure of healthcare professional to SARS-CoV2 virus during the phlebotomy procedure during the earlier phase of the pandemic when less was known about the virus.

\section{Limitation}

The major proportion of laboratory test ordering was received for inpatients with only a minor proportion of outpatients during the prepandemic phase. While during the pandemic, the tests ordered were for inpatients only. Thus, the laboratory census during the prepandemic period includes both the inpatient and outpatient records. Due to logistic issues, the number of laboratory tests ordered and BCT used for the outpatients (however low) during this period could not be excluded and therefore is a confounding factor. Also, the overall laboratory utilization and hospital bed utilization observed in this study may vary at different time points and in different centers depending on the local incidence of COVID-19 infection.

\section{Conclusion}

There was an overall decline in the laboratory utilization during the pandemic period. Logistics related to introduction of new test panel, new instrumentation, staff training, and time lags due to repeated nationwide or local lockdown can result in delays in test operations and need extra attention for providing uninterrupted quality patient service. Understanding and correlating the trends with hospital bed utilization also proved beneficial and can help to maximize the productivity of the laboratory by minimizing wastage and can help in better preparedness for the challenges imposed during similar exigencies.

\section{Conflict of Interest}

None.

\section{References}

1 Lippi G, Plebani M. The critical role of laboratory medicine during coronavirus disease 2019 (COVID-19) and other viral outbreaks. Clin Chem Lab Med 2020;58(07):1063-1069

2 Soto AB. The role of laboratory medicine specialists in the COVID19 pandemic. Advances in Laboratory Medicine/Avances en Medicina de Laboratorio 2020;1(2)

3 Cumulative of the coronavirus (COVID-19) confirmed, recovered and deceased numbers across India. 2021. Accessed on January 15 , 2021, at: statista.com/statistics/1104054/india-coronaviruscovid-19-daily-confirmed-recovered-death-cases

4 Durant TJS, Peaper DR, Ferguson D, Schulz WL. Impact of COVID19 pandemic on laboratory utilization. J Appl Lab Med 2020;5 (06):1194-1205

5 Ongen-Ipek B, Sitar ME, Karadeniz A. Adaptation of clinical laboratories to COVID 19 pandemic: changes in test panels, overcoming problems and preparation suggestions for future pandemics adaptation of clinical laboratories to COVID 19 pandemic. Clin Lab 2020;66(11):

6 Ali N. Elevated level of C-reactive protein may be an early marker to predict risk for severity of COVID-19. J Med Virol 2020;92(11): 2409-2411

7 Wang L. C-reactive protein levels in the early stage of COVID-19. Med Mal Infect 2020;50(04):332-334

8 Huang I, Pranata R, Lim MA, Oehadian A, Alisjahbana B. C-reactive protein, procalcitonin, $\mathrm{D}$-dimer, and ferritin in severe coronavirus disease-2019: a meta-analysis. Ther Adv Respir Dis 2020; 14:1753466620937175https://doi.org/ $10.1177 / 1753466620937175$

9 McCoy H, Kenney MA. Magnesium and immune function: recent findings. Magnes Res 1992;5(04):281-293

10 Iotti S, Wolf F, Mazur A, Maier JA. The COVID-19 pandemic: is there a role for magnesium? Hypotheses and perspectives. Magnes Res 2020;33(02):21-27

11 Yao Y, Cao J, Wang Q, et al. D-dimer as a biomarker for disease severity and mortality in COVID-19 patients: a case control study. J Intensive Care 2020;8:49

12 Zhang L, Yan X, Fan Q et al. D-dimer levels on admission to predict in-hospital mortality in patients with Covid-19. J Thromb Haemost 2020;18(06):1324-1329 
13 Yu HH, Qin C, Chen M, Wang W, Tian DS. D-dimer level is associated with the severity of COVID-19. Thromb Res 2020; 195:219-225

14 Lin Z, Long F, Yang Y, Chen X, Xu L, Yang M. Serum ferritin as an independent risk factor for severity in COVID-19 patients. J Infect 2020;81(04):647-679

15 Li C, Ye J, Chen Q, et al. Elevated lactate dehydrogenase (LDH) level as an independent risk factor for the severity and mortality of COVID-19. Aging (Albany NY) 2020;12(15): 15670-15681

16 Chen LYC, Hoiland RL, Stukas S, Wellington CL, Sekhon MS. Assessing the importance of interleukin-6 in COVID-19. Lancet Respir Med 2021;9(02):e13
17 Drews RE. Critical issues in hematology: anemia, thrombocytopenia, coagulopathy, and blood product transfusions in critically ill patients. Clin Chest Med 2003;24(04):607-622

18 Loh TP, Horvath AR, Wang CB, et al; International Federation of Clinical Chemistry and Laboratory Medicine Taskforce on COVID19. Laboratory practices to mitigate biohazard risks during the COVID-19 outbreak: an IFCC global survey. Clin Chem Lab Med 2020;58(09):1433-1440

19 Loh TP, Horvath AR, Wang CB, et al; International Federation of Clinical Chemistry and Laboratory Medicine Taskforce on COVID19. Operational considerations and challenges of biochemistry laboratories during the COVID-19 outbreak: an IFCC global survey. Clin Chem Lab Med 2020;58(09):1441-1449 